\title{
Penile vascular diagnostic categorization using penile duplex Doppler ultrasound: Differences in vascular hemodynamics parameters by differences in anatomic sampling location
}

\author{
Fabio Pezzoni ${ }^{1}$, Fabrizio Ildefonso Scroppo ${ }^{2}$ \\ ${ }^{1}$ Vascular Surgeon, University of Milano, Italy-member of SIA, Italy; \\ ${ }^{2}$ Department of Urology and Andrology, UOC Urology Ospedale di Circolo, Varese, Italy.
}

\begin{abstract}
Summary Objectives. In 2013 the International Society for Sexual Medicine(ISSM) published the guidelines regarding the standard operating procedure (SOP) for penile duplex Doppler ultrasound (PDDU). Although ISSM-SOP have given important strides in reducing interobserver variability in PDDU by procedural protocol and parameters these guidelines do not address the anatomic location along the penis at which hemodynamic measurements have to be done. In our opinion a "double sampling" may be interesting to detect the arteriogenic or venogenic nature of the erectile dysfunction (ED). In particular sampling measurements at the "crus" (at the level of the peno-scrotal junction) may be significative for detection of veno-occlusive dysfunction (VOD), whereas an evaluation at "mid penis" (1/2 distance between peno-scrotal junction and coronal sulcus), may be useful to diagnose an arterial insufficiency (AI).

Material and Methods. We evalued 90 men, mean age 56.3, affected with ED of medium degree, responder to PDE5-I that urdergone to PDDU and also responder after pharmacologic intracavernosal injection (PII) of prostaglandin E1 $20 \mathrm{mcg}$, with rigid erection and normal maintenance. We moreover evalued 90 men in youthful age (mean 35.2), in absence of vascular risk factors, no responder to PDE5-I that undergone to PDDU by PII at high dosage (bimix:

prostaglandin E1 $20 \mathrm{mcg}$, papaverine $20 \mathrm{mg}$ ).

Results. In the first pool the sampling at "mid penis" resulted significative for arterial insuffciency (AI) in $81 \%$ (73), in presence of normal or borderline end diastolic velocity (EDV).

Sampling at the "crus" resulted negative for VOD in $90 \%$ (81). In the second pool, $66.6 \%$ (60) resulted responder

with rigid erection and normal maintenance in presence of normal hemodynamic parameters: peak systolic velocity (PSV) and end diastolic velocity (EDV) both at the "crus" and at "mid penis" sampling. $33.4 \%$ (30) responded with a semirigid erection and manifested a constant deficit of maintenance; at the "crus" and at "mid penis" the hemodynamic arterial parameters resulted normal. At the "crus" the EDV resulted signifcantly augmented (VOD index) in 96.6\% (29); at "mid penis" augmented EDV was founded in 50\% (15).

Conclusions. These observational data would be able to confirm the utility of a routinary "double sampling" procedure, at the "crus" and at "md penis", during PDDU in order to better distinguish between VOD or AI or in any case to be useful to stimulate a future more precise standardization in execution of PDDU examination.
\end{abstract}

KEY WORDS: Duplex Doppler ultrasonography; Erectile dysfunction; Penile venous leakage; Arteriogenic impotence.

Submitted 14 January 2016; Accepted 21 February 2016

\section{INTRODUCTION}

Erectile dysfunction (ED), together with premature ejaculation, is the most common male sexual disorder. Vascular ED comprehends cavernosal artery insufficiency and dysfunction of penile veno-occlusive system. These two mechanisms frequently cohabit in a single patient, representing two different steps of the same disease. It is demonstrated that around $70 \%$ of all ED is related to pathological penile vasculature either through reduced inflow or augmented outflow (1). The recent American Urological Association (AUA) guidelines on ED counsel, in selected patients, a second level vascular diagnostic evaluation $(2,3)$. The classic test consists in penile duplex Doppler ultrasound (PDDU) after pharmacologic intracavernosal injection (PII) inducing erection, to discriminate arterial insufficiency (AI) and venoocclusive dysfunction (VOD) from other causes of ED. In 2013 the International Society for Sexual Medicine (ISSM) published the guideline regarding the standard operating procedure (SOP) for PDDU (4). Although ISSM (SOP) have given important strides in reducing interobserver variability in PDDU by precise procedural protocols and parameters, these guidelines do not address the anatomic location along the penis at which

hemodynamic measurements have to be done, during the execution of the examination. PDDU is aimed at the functional and anatomical study of the cavernosal arterial inflow. When vascular evaluation is indicated PDDU offers the least invasive and accurate data to assess the penile arterial inflow. The parameters to estabilish the integrity of the arterial flux are: peak systolic flow velocity (PSV), cavernous arterial diameters, acceleration time. PDDU also can values the cavernous veno-occlusive mechanism in the post-injection phase, considering the end-dyastolic velocity (EDV) and the resistance index (RI); persisting diastolic blood flow and/or low RI: suspect of VOD. A recent study by Pagano has pointed out the importance of the differences in vascular hemodynamics parameters by differences in anatomic sampling location of ultrasound probe placement for cavernosal arteries during dynamic PDDU (5). In our opinion a "double sampling" may be interesting to detect the arteriogenic or venogenic 


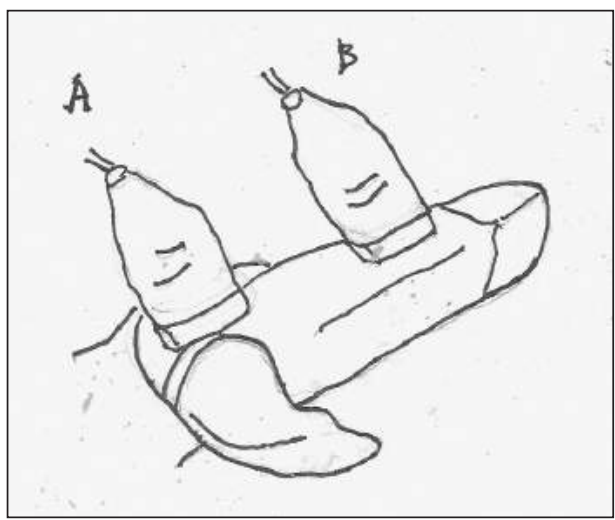

Figure 1.

Crus and

mid penis. nature of the vasculogenic ED $(6,7)$. In particular sampling measurements "at the crus" (at the level of the penoscrotal junction) may be significatives for detection of VOD, whereas an evaluation at "mid penis" (1/2 distance between penoscrotal junction and coronal solcus), may be useful to diagnose an arteriogenic insufficiency (Figure 1).

\section{Material AND METHOdS}

We evalued a total of 90 men, mean age 56.3, affected with persistent ED of medium degree (IIEF score between 10 and 15), in absence of Peyronie's disease, responder to PDE5-inhibitors that undergone to PDDU and also responder after PII with rigid erection and normal maintenance (8).

According to the International Consultation on Sexual Dysfunction, ED is defined as "persistent" when there is inhability to attain and mantain an erection sufficient to permit satisfactory sexual performance and the symptoms have to persist for a minimum duration of six months. They took PDE5-inhibitors (vardenafil or tadalafil) at personalized dosage after an evaluation "case for case" based on: clinical factors, age, frequency of sexual intercourse, life style. PDDU assessments were done using a $12 \mathrm{MHz}$ linear array transducer, after PII of prostaglandin E1 20 mcg. Parameters that have been considered for a vascular diagnosis were PSV, EDV, acceleration time and RI. PSV was considered normal for mean values $>30 \mathrm{~cm} / \mathrm{sec}$.; EDV for values $<5 \mathrm{~cm}$./sec., acceleration time for values $<1 \mathrm{sec}$. To reach PSV, and RIhad to be $>0.80$, according to ISSM (SOP) (4). We moreover evalued 90 men in youthful age, mean 35. 2, in absence of vascular risk factors, no responder to PDE5-inhibitors (vardenafil e/o tadalafil) in satisfactory way, also at maximum dosage. They undergone to dynamic PDDU by PII at high dosage of "bimix": prostaglandin El $20 \mathrm{mcg}$. + papaverine $20 \mathrm{mg}$.

The same PDDU vascular parameters were also analyzed in this group. In all the cases the examinations were performed by a "double sampling" measurements positioning the probe "at the crus" (at the level of the penoscrotal junction) and at "mid penis" ( $1 / 2$ distance between penoscrotal junction and coronal solcus) with the aim to postulate the arteriogenic or venogenic nature of the vasculogenic ED.

\section{RESULTS}

The sampling, in the first group of men at "mid penis" resulted significative for presence of $\mathrm{AI}$ in $81 \%$ (73): the mean value of PSV was $25.3 \mathrm{~cm} / \mathrm{sec}$. (Figure 2). EDV resulted normal or borderline in all the patients (mean $2.1 \mathrm{~cm} / \mathrm{sec}$.), acceleration time resulted significative for presence of angiosclerosis in all the men (mean $1.5 \mathrm{sec}$. ), RI had a mean normal value of 0.92 (9) (Figure 3).

Sampling at the "crus" resulted negative for DVO in $90 \%$ (81): mean EDV resulted $2.5 \mathrm{~cm} / \mathrm{sec}$. An EDV value $>5$ $\mathrm{cm} / \mathrm{sec}$. is considered by ISSM-(SOP) a significative DVO presence index (9) (Table 1).

In the second group of men $66.6 \%$ (60) resulted responder with rigid erection and normal maintenance in presence of normal hemodynamic parameters: PSV, EDV, RI both at the "crus" and at "mid penis" sampling (10). $33.4 \%$ (30) responded with a semirigid erection and

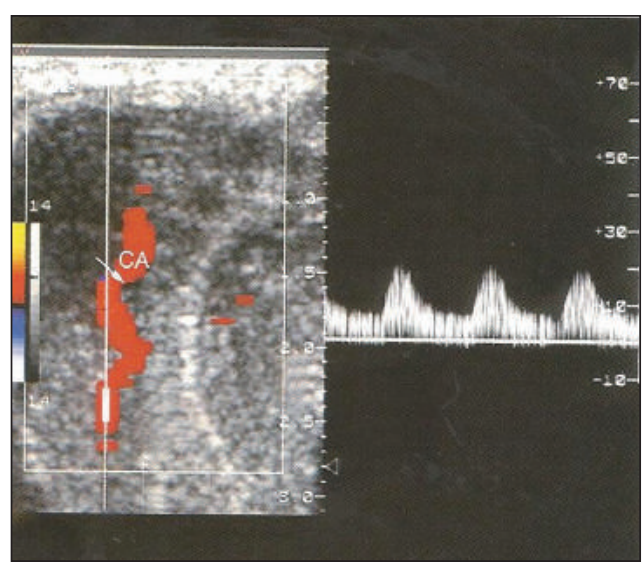

Figure 2. Arteriogenic insufficiency.

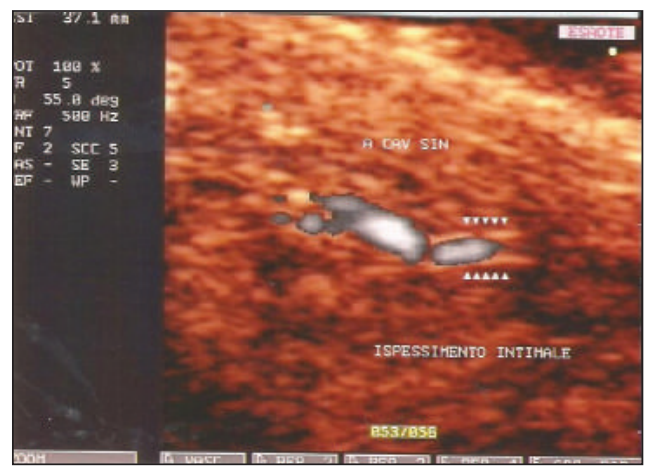

Figure 3. Angiosclerosis.

Table 1.

First pool of patients (90).

$\begin{array}{ll}\text { Sampling "mid penis" } & \text { Sampling at the "crus" } \\ \text { Positive for Al: } \mathbf{8 1} \% \mathbf{( 7 3 )} & \text { Negative for VOD: } \mathbf{9 0 \% ( 8 1 )} \\ \text { EDV: normal/borderline 100\% (90) } & \end{array}$

manifested a constant deficit of maintenance; at the "crus" and at "mid penis" the hemodynamic arterial parameters resulted normal (Figures 4, 5).

At the "crus" EDV resulted significantly augmented (DVO index) in $96.6 \%$ (29) with a mean value of $7.8 \mathrm{~cm} / \mathrm{sec}$; RI also had a DVO significative mean value of 0.71 . At "mid penis" augmented EDV was founded in 50\% (15) (11) (Figure 6) (Table 2). 


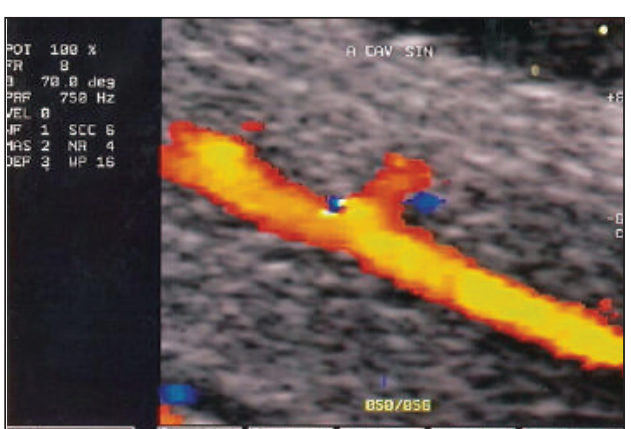

Figure 4. Normal cavernous artery.

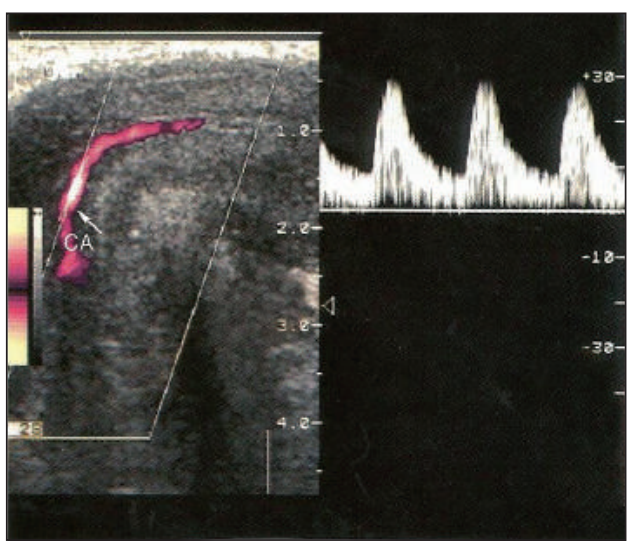

Figure 5.

Normal

arterial

parameters.

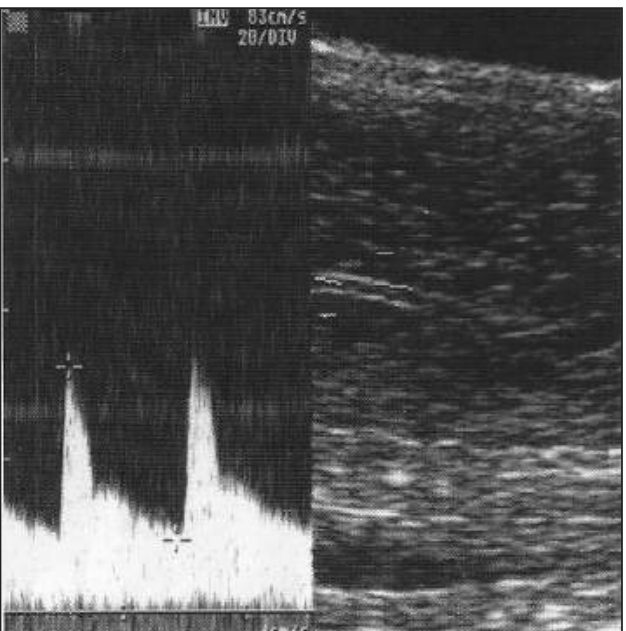

Figure 6.

Edv

augmented (DVO).

\section{Table 2.}

Second pool of patients (90).

\begin{tabular}{|ll}
\hline Sampling at the "crus" & Sampling "mid penis" \\
Positive for VOD: $\mathbf{9 6 . 6 \% ~ ( 2 9 )}$ & Negative for Al: $100 \%(90)$ \\
Negative for Al 100\% (90) &
\end{tabular}

\section{DiscuSSION AND CONCLUSIONS}

VOD is not classified in a precise nosologycal condition, but like a multifactorial ethiology syndrome and the the "venous-leakage" constitues a complex fenomenon interesting structural abnormalities in corpora cavernosa and/or in the tunica albuginea. However these oservational data would be able to confirm the utility of a routinary "double sampling" procedure (at the "crus" and at "mid penis") during PDDU in order to better distinguish between venogenic or arteriogenic vasculogenic disorders or in any case to be useful to stimulate a future more precise standardization in execution of PDDU examination.

\section{REFERENCES}

1. Goldstein I. Male sexual circuitry, working group for the study of central mechanisms in erectile dysfunction. Sci Am. 2000; 2803:70-5.

2. American Urological A. (AUA guideline. Management of erectile dysfunction). Arch Esp Urol. 2011; 64:4.

3. Board of Directors, American Urological Association AUA. Policy Statement:Diagnostic evaluation of erectile dysfunction, May 2012 (revised). https://www. auanet. org/education/policy-statements/evaluations-of-erectile-dysfunction. cfm (accessed April 1, 2015).

4. Sikka SC, Hellstrom WJ, Brock G, Morales AM. Standardization of vascular assessment of erectile dysfunction: Standardoperating procedures for duplex ultrasound. J Sex Med. 2013; 10:120-9.

5. Pagano J, Stahal PJ. Variation in penile hemodynamics by anatomic location of cavernosal artery imaging in penile duplex Doppler ultrasound. J Sex Med. 2015; 12:1911-1919.

6. Furlow WL, Knoll LD. Arteriogenic impotence. Diagnoses and management. Problems in Urology 1991; 5:577-93.

7. Virag R, Zwang G, Dermange H. Vasculogenic impotence: A review of 92 cases with 54 surgical operations. Vasc Surg. 1981; 15:9.

8. Rosen RC, Riley A, Wagner G, et al. The international index of erectile dysfunction (IIEF): a multidimensional scale of assessment of erectile dysfunction. Urology 1997; 49:822-30.

9. Kim SH, Paick JS, Lee SE, et al. Doppler sonography of deep cavernosal artery of the penis: variation of peak systolic velocity according to sampling location. J Ultrasound Med. 1994; 13:591-4.

10. Baumhakel M, Schlimmer N, Kratz M, et al. Cardiovascular risk, drugs and erectile function: a systematic analysis. Int J Clin Pract. 2011; 65:289-98.

11. Kropman RF, Schipper J, van Oostayen JA, et al. The value of increased end diastolic velocity during penile duplx sonography in relation to pathological venous leakage in erectle dysfunction.J Urol. 1992; 148:314-7.

\section{Correspondence}

Fabio Pezzoni (Corresponding Author)

Tel. ++39.333 .40 .36 .098$

fabiopezzonil@alice.it

Vascular Surgeon, University of Milano

V. G. Pezzotti, 36 - Milano

Scroppo Fabrizio I., MD Member of SIA

fabrizio.scroppo@ospedale.varese.it

Department of Urology and Andrology,UOC Urology Ospedale di Circolo, Viale L. Borri, 57 - Varese, Italy 